\title{
EXPONENT BOUNDS FOR A CONVOLUTION INEQUALITY IN EUCLIDEAN SPACE WITH APPLICATIONS TO THE NAVIER-STOKES EQUATIONS
}

\author{
CHRIS ORUM AND MINA OSSIANDER \\ (Communicated by Walter Craig)
}

\begin{abstract}
The convolution inequality $h * h(\xi) \leq B|\xi|^{\theta} h(\xi)$ defined on $\mathbf{R}^{n}$ arises from a probabilistic representation of solutions of the $n$-dimensional Navier-Stokes equations, $n \geq 2$. Using a chaining argument, we establish in all dimensions $n \geq 1$ the nonexistence of strictly positive fully supported solutions of this inequality for $\theta \geq n / 2$. We use this result to describe a chain of continuous embeddings from spaces associated with probabilistic solutions to the spaces $B M O^{-1}$ and $B M O_{T}^{-1}$ associated with the Koch-Tataru solutions of the Navier-Stokes equations.
\end{abstract}

\section{INTRODUCTION}

Convolution inequalities of the form

$$
h * h(\xi)=\int_{\mathbf{R}^{n}} h(\xi-\eta) h(\eta) d \eta \leq B|\xi|^{\theta} h(\xi), \quad \xi \in \mathbf{R}^{n}, \quad \theta \geq 0, \quad B>0,
$$

arise in the analysis of the incompressible Navier-Stokes equations via probabilistic representations of solutions. Our main theorem shows that if $h: \mathbf{R}^{n} \rightarrow(0, \infty]$ is a fully supported function satisfying (1), then the range of the exponent $\theta$ is constrained by the dimension $n$. Letting $\mathcal{H}^{\theta}\left(\mathbf{R}^{n}\right)$ denote the class of solutions of (11) on $\mathbf{R}^{n}$ we obtain:

Theorem 1. If $h \in \mathcal{H}^{\theta}\left(\mathbf{R}^{n}\right), n \geq 1$, then $\theta<n / 2$.

Our study of this inequality is motivated by an effort to better understand the structure and limitations of the stochastic cascade representation of solutions to the Navier-Stokes equations as first introduced by Le Jan and Sznitman [14] and then extended by later authors. Essentially, any $h$ satisfying (11) induces a Banach space $\mathcal{F}_{h}$ (the initial value space) and another space $\mathcal{F}_{h, T}=B\left(0, T ; \mathcal{F}_{h}\right)$ of bounded $\mathcal{F}_{h^{-}}$ valued functions defined on $[0, T]$ (the path space) that supports a Picard iteration scheme for establishing existence and uniqueness of solutions of the Navier-Stokes initial value problem. The function $h$, which we refer to as a majorizing kernel,

Received by the editors January 13, 2011 and, in revised form, January 22, 2012.

2010 Mathematics Subject Classification. Primary 35Q30, 76D05, 42B37; Secondary 76M35, 39B72, 60J80.

This work was partially supported by the U.S. National Science Foundation through the Focussed Research Group collaborative awards DMS-0073958 and DMS-0940249. 
must be fully supported to correspond to real-valued solutions. Additionally, if the particular $h$ inducing $\mathcal{F}_{h}$ has $\theta=1$, then the solutions so obtained are global in time under the restriction that the data are sufficiently small. If $h$ has $0 \leq \theta<1$, then the Picard iteration scheme accommodates arbitrarily large data, but the solutions are restricted to being local in time; i.e. take values in $\mathcal{F}_{h, T}$ where $T$ depends on the size of the initial datum in the $\mathcal{F}_{h}$-norm. In the case $\theta=1$ there is an equivalent stochastic cascade model providing solutions to the Cauchy problem operative for all $t \geq 0$.

The authors of [3] give examples of majorizing kernels and analyze some properties of classes of majorizing kernels. In all cases considered however the fully supported examples with exponent $\theta=1$ are in dimensions $n \geq 3$. The results presented here provide further understanding of this phenomena by demonstrating that there are no fully supported solutions with $\theta=1$ in $\mathbf{R}^{2}$. Correspondingly, there is no direct analogue of the global 3-dimensional stochastic cascades model in dimension $n=2$. On the other hand, the Picard iteration method applied with $\mathcal{F}_{h, T}, 0 \leq \theta<1$, is sufficient to show the existence of classes of solutions that are local in time in any dimension $n \geq 2$. In other words, Theorem 1 imposes no limitations on these local solutions (in any dimension $n \geq 2$ ); it only limits the approach for global solutions in dimension $n=2$.

The organization of this paper is as follows. Section 2 reviews the origins and importance of the convolution inequality (11). Section 3 contains a short proof of Theorem 1. In Section 4 we consider the continuous embeddings of certain $\mathcal{F}_{h}$ into the pseudomeasure spaces $P M^{n-\theta}$, which Theorem 1 plays a role in establishing, as well as successive embeddings into Besov spaces and the spaces $B M O^{-1}$ and $B M O_{T}^{-1}$ associated with the Koch-Tataru solutions.

\section{BACKGROUND AND MOTIVATION}

Consider the incompressible Navier-Stokes equations formulated as a Cauchy problem on all of $\mathbf{R}^{n}$ where $n \geq 2$. This system models the flow of an idealized incompressible viscous fluid issued from an initial velocity field $u_{0}=u_{0}(x)$ at time $t=0$. Dimension $n=3$ is of central importance, but the formulation is of interest in arbitrary dimension $n \geq 2$. The unknowns are the velocity vector $u=u(x, t)=\left(u_{i}(x, t)\right)_{i=1}^{n}$ and scalar pressure $p=p(x, t)$, where $x=\left(x_{1}, \ldots, x_{n}\right)$. The system consists of $n+1$ coupled nonlinear equations

$$
\begin{aligned}
& \frac{\partial u_{i}}{\partial t}(x, t)+\sum_{j=1}^{n} u_{j}(x, t) \frac{\partial u_{i}}{\partial x_{j}}(x, t)=\nu \sum_{j=1}^{n} \frac{\partial^{2} u_{i}}{\partial x_{j}^{2}}(x, t)-\frac{\partial p}{\partial x_{i}}(x, t)+g_{i}(x, t), \\
& \sum_{j=1}^{n} \frac{\partial u_{j}}{\partial x_{j}}(x, t)=0
\end{aligned}
$$

supplemented by the initial condition $\lim _{t \rightarrow 0} u(x, t)=u_{0}(x)$. Here $\nu$ denotes the kinematic viscosity and $g(x, t)=\left(g_{i}(x, t)\right)_{i=1}^{n}$ is an external forcing term. For simplicity we may assume that $\nabla \cdot u_{0}(x)=0$ and $\nabla \cdot g(x, t)=0$ for all $t$.

In 1997 Le Jan and Sznitman [14] introduced a representation of the solutions of a Fourier space integral formulation of (2) in three spatial dimensions as a multiplicative functional defined on a continuous-time branching process. These Fourier 
transformed Navier-Stokes equations (FNS) may be written

$$
\begin{aligned}
\hat{u}(\xi, t)= & e^{-\nu|\xi|^{2} t} \hat{u}_{0}(\xi)+\int_{0}^{t} e^{-\nu|\xi|^{2}(t-s)} \hat{g}(\xi, s) d s \\
& +(2 \pi)^{-n / 2} \int_{0}^{t}|\xi| e^{-\nu|\xi|^{2}(t-s)} \int_{\mathbf{R}^{n}}\left[-\mathrm{i} \frac{\xi}{|\xi|} \cdot \hat{u}(\eta, s)\right] \hat{\mathbf{P}}(\xi) \hat{u}(\xi-\eta, s) d \eta d s,
\end{aligned}
$$

where $\xi=\left(\xi_{1}, \ldots, \xi_{n}\right)$ is the Fourier space variable, $\hat{u}(\xi, t)$ denotes the spatial Fourier transform of the unknown velocity field (and similarly for $\hat{g}(\xi, t)$ and $\hat{u}_{0}(\xi)$ ), and $\hat{\mathbf{P}}(\xi)$ denotes the Leray-Helmholtz projection whose pointwise action in Fourier space is to project a vector $z \in \mathbf{C}^{n}$ onto the subspace orthogonal to $\xi \neq 0$ :

$$
\hat{\mathbf{P}}(\xi) z=z-\left(\mathbf{e}_{\xi} \cdot z\right) \mathbf{e}_{\xi} ; \quad \mathbf{e}_{\xi}=\frac{\xi}{|\xi|} .
$$

A key device in the $\mathbf{R}^{3}$ representation in [14] is the dimension specific rescaling of $\hat{u}$ and $\hat{g}$,

$$
\chi(\xi, t)=\frac{2}{\nu}\left(\frac{\pi}{2}\right)^{3 / 2}|\xi|^{2} \hat{u}(\xi, t), \quad \varphi(\xi, t)=\frac{4}{\nu^{2}}\left(\frac{\pi}{2}\right)^{3 / 2} \hat{g}(\xi, t),
$$

which allows the simultaneous description of a 'splitting distribution' for a pair of particles $\left\{\Xi_{1}, \Xi_{2}\right\}$ replacing $\xi$ in the branching process, namely

$$
\operatorname{Pr}\left(\Xi_{i} \in A\right)=\frac{1}{\pi^{3}} \int_{A} \frac{|\xi|}{|\xi-\eta|^{2}|\eta|^{2}} d \eta, \quad A \subseteq \mathbf{R}^{3}, \quad i=1,2, \quad \Xi_{1}+\Xi_{2}=\xi,
$$

and the normalization of $|\xi| \exp \left\{-\nu|\xi|^{2} s\right\}$ to the density of an exponential random variable describing the random lifetime of the particle of type $\xi$ so replaced.

Details of this construction may be found in [14, 3]; extensions may be found in [4, [5], [9, 10], 18], 19]. Related analytical papers are [2], 12], 20], 21], 22]. Essentially the solution is represented in the form of an expected value

$$
\hat{u}(\xi, t)=h(\xi) \mathbf{E X}(\xi, t),
$$

where $h(\xi)=\pi^{-3}|\xi|^{-2}$ solves the convolution equation $h * h(\xi)=|\xi| h(\xi)$ on $\mathbf{R}^{3}$ and $\mathrm{X}(\xi, t)$ is defined by a backward recursion arising from a probabilistic interpretation of the rescaled formulation of (3). Figure1illustrates the branching process and the construction of the multiplicative functional $\mathbf{X}(\xi, t)$. The $\otimes_{\xi}$-operation performed at each of the binary nodes in the branching process encodes the algebraic structure of the bilinear term on the right-hand side of (3): for two vectors $z, w \in \mathbf{C}^{3}$ we define $z \otimes_{\xi} w \in \mathbf{C}^{3}$ by $z \otimes_{\xi} w=-\mathrm{i}\left[z \cdot \mathbf{e}_{\xi}\right] \hat{\mathbf{P}}(\xi) w$.

This representation provides existence and uniqueness results for the solutions of (3) in the space of pseudomeasures $\left(P M^{2}\right)^{3}$. The scale of pseudomeasure spaces is defined by

$$
P M^{a}=\left\{f \in \mathcal{S}^{\prime}\left(\mathbf{R}^{n}\right): \hat{f} \in L_{\text {loc }}^{1}\left(\mathbf{R}^{n}\right),\left\|f ; P M^{a}\right\|=\underset{\xi \in \mathbf{R}^{n}}{\operatorname{esssup}}|\xi|^{a}|\hat{f}(\xi)|<\infty\right\},
$$

where $a \geq 0$ is a given parameter and $\mathcal{S}^{\prime}\left(\mathbf{R}^{n}\right)$ denotes the space temperate distributions on $\mathbf{R}^{n}$. Alternatively, the spaces $P M^{a}$ may be regarded as homogeneous Besov-type spaces based on the classical space of pseudomeasures $P M=P M^{0}$ :

$$
P M^{a}=\dot{B}_{P M}^{a, \infty}=\left\{f \in \mathcal{S}^{\prime}\left(\mathbf{R}^{n}\right): \sup _{j \in \mathbf{Z}} 2^{a j}\left\|\Delta_{j} f\right\|_{P M}<\infty\right\} .
$$

Here $\Delta_{j} f$ is the $j$ th dyadic block of the Littlewood-Paley decomposition of $f$. 


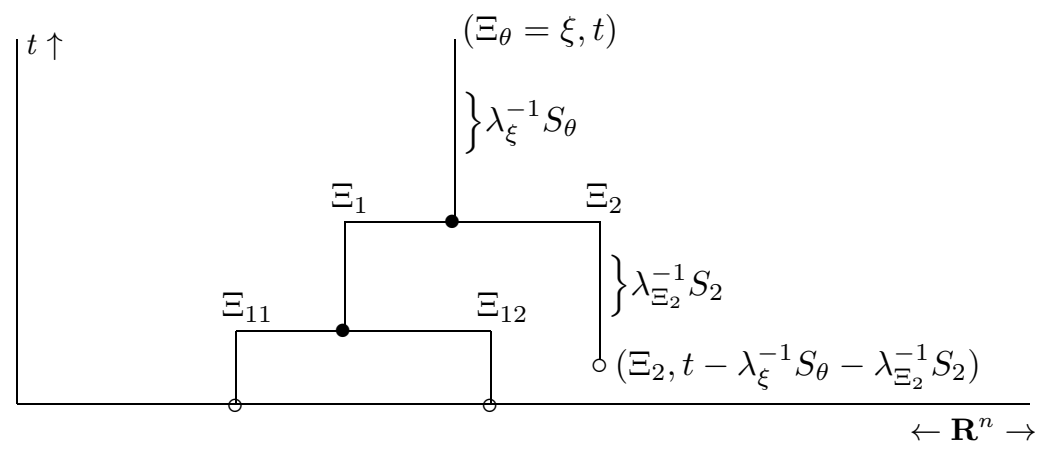

Figure 1. A schematic illustration of the branching process and the construction of $X(\xi, t)$ : a particle of type $\xi=\Xi_{\theta}$ lives for a random length of time $\lambda_{\xi}^{-1} S_{\theta}$ and then dies out. Depending on the outcome of a Bernoulli random variable with mean $1 / 2$, it is either not replaced at all or replaced by two correlated particles $\Xi_{1}$ and $\Xi_{2}$ distributed as (4), or more generally (8). The two new particles in turn live for independent random lifetimes, and so the process continues. There are two types of nodes: input nodes (o) accept data when a particle dies out without replacement or when its lifetime extends below the horizontal axis at time $t=0$. Operational nodes $(\bullet)$ combine data according to $z, w \mapsto m\left(\Xi_{\mathbf{v}}\right) z \otimes_{\Xi_{\mathbf{v}}} w$ and send the output upward. Here $m(\xi)$ is a multiplicative factor that arises from the rescaling of $\hat{u}$ by $h, \lambda_{\xi}=\nu|\xi|^{2}, \mathbf{v} \in\{\theta, 1,2,11, \ldots\}$, and $S_{\theta}, S_{1}, S_{2}, \ldots$ are $i . i . d$. standard exponential random variables.

Returning to the stochastic cascade, it was later recognized [6] that the same existence and uniqueness results could be obtained by applying the Picard iteration argument with the Banach space $B\left(0, T ;\left(P M^{2}\right)^{3}\right)$ of bounded functions $f(t):[0, T] \rightarrow\left(P M^{2}\right)^{3}$. This argument is notable for the continuity of

$$
\mathbf{B}=\mathbf{B}(u, v)(x, t)=\int_{0}^{t} e^{\nu(t-s) \Delta} \mathbf{P} \nabla \cdot(u \otimes v)(s) d s .
$$

That is, $\mathbf{B}: E \times E \rightarrow E$ is continuous if $E=B\left(0, T ;\left(P M^{2}\right)^{3}\right)$ but is not continuous in general, in which case the Picard iteration argument typically requires the use of an embedded subspace with a second norm; see e.g. [1, p. 220], [7, [15], 16].

The authors of [3] generalize this approach by showing that the $h$ in (5) may belong to a more general class of FNS majorizing kernels which are positive solutions of (1) parameterized by the exponent $\theta$. There are two natural Banach spaces associated with a given majorizing kernel. The first is the majorization space

$$
\mathcal{F}_{h}=\left\{f \in \mathcal{S}^{\prime}\left(\mathbf{R}^{n}\right)^{n}: \hat{f}(\xi) \in L_{\mathrm{loc}}^{1}\left(\mathbf{R}^{n}\right)^{n},\left\|f ; \mathcal{F}_{h}\right\|=\sup _{\xi \in \mathbf{R}^{n}}[h(\xi)]^{-1}|\hat{f}(\xi)|<\infty\right\} .
$$

The initial data for the Cauchy problem belongs to this space. The second is the path space $\mathcal{F}_{h, T}=B\left(0, T ; \mathcal{F}_{h}\right)$ of bounded $\mathcal{F}_{h}$-valued functions defined on the interval $[0, T]$ with norm

$$
\left\|f(t) ; \mathcal{F}_{h, T}\right\|=\sup _{0 \leq t \leq T}\left\|f(t) ; \mathcal{F}_{h}\right\| .
$$


The solutions belong to this space. It turns out that $\mathbf{B}: \mathcal{F}_{h, T} \times \mathcal{F}_{h, T} \rightarrow \mathcal{F}_{h, T}$ is again continuous for any majorizing kernel of exponent $0 \leq \theta \leq 1$, and the Picard iteration argument is directly applicable without the introduction of a second norm. This yields global existence and uniqueness results in the case $\theta=1$ (with small data) and local existence and uniqueness results in the case $0 \leq \theta<1$ (with arbitrarily large data). The argument works for the FNS equations formulated in any dimensions $n \geq 2$ subject to the constraint $\theta<n / 2$ of Theorem 1 ,

For majorizing kernels with exponent $\theta=1$, the Picard iteration scheme can be connected to stochastic cascades as follows. A solution $h$ of (11) provides the following splitting distribution for a branching process generalizing (4):

$$
\operatorname{Pr}\left(\Xi_{1} \in A\right)=\int_{A} \frac{h(\xi-\eta) h(\eta)}{h * h(\xi)} d \eta, \quad A \subseteq \mathbf{R}^{n}, \quad \Xi_{1}+\Xi_{2}=\xi .
$$

This specializes to (4) in dimension $n=3$ with $h(\xi)=\pi^{-3}|\xi|^{-2}$. For the more general branching processes and $\mathrm{X}(\xi, t)$ defined accordingly, one can define a sequence of events $\left\{G_{k}\right\}_{k \geq 0}$ pertaining to the branching process so that the sequence $\mathbf{E}\left(\mathrm{X}(\xi, t) ; G_{k}\right)$ and the iterates of the Picard contraction argument are in one-toone correspondence. This is established in [3] with the conclusion that for global solutions with $\theta=1$, the existence of the expected value representation and the convergence of the Picard iteration scheme are essentially equivalent.

\section{MAIN THEOREM}

The majorizing kernels considered in 3 are allowed to be supported on various convex additive semigroups $W \subset \mathbf{R}^{n}$. Here however we focus entirely on fully supported majorizing kernels: those $h$ with $\int_{A} h(\xi) d \xi>0$ for all subsets $A \subseteq \mathbf{R}^{n}$ having positive Lebesgue measure. Fully supported majorizing kernels correspond to majorization spaces $\mathcal{F}_{h}$ and $\mathcal{F}_{h, T}$ that contain real data and solutions.

Definition 2. A majorizing kernel with exponent $\theta$ is a tempered function (a tempered distribution that is also a function) $h: \mathbf{R}^{n} \rightarrow(0, \infty]$ satisfying the following conditions:

(i) $h * h(\xi) \leq B|\xi|^{\theta} h(\xi)$ for all $\xi \in \mathbf{R}^{n}$ with constants $B>0$ and $\theta \geq 0$;

(ii) $h^{-1}(\infty)$ has $n$-dimensional Lebesgue measure zero.

We set $\Omega_{h}=\mathbf{R}^{n} \backslash h^{-1}(\infty)$. If $B$ is $\operatorname{such}$ that $\sup _{\xi} h * h(\xi) /|\xi|^{\theta} h(\xi)=B$, then $B$ is sharp. If $B=1$ is sharp, then $h$ is standardized.

The set of majorizing kernels of exponent $\theta$ defined on $\mathbf{R}^{n}$ is denoted $\mathcal{H}^{\theta}\left(\mathbf{R}^{n}\right)$. It is possible for a given majorizing kernel to have a range of exponents.

The invertible map $h \mapsto B^{-1} h$ on $\mathcal{H}^{\theta}\left(\mathbf{R}^{n}\right)$ has the effect of standardizing any nonstandardized majorizing kernel with sharp constant $B$; hence for the purpose of proving Theorem 1 we assume, without loss of generality, that $h$ is standardized. We also make this assumption in the proofs of the lemmas in this section.

Lemma 3. Let $h \in \mathcal{H}^{\theta}\left(\mathbf{R}^{n}\right), \theta \geq 0$. Then for all $R>0, h(\xi)$ is bounded away from zero on the closed ball $\bar{B}(0, R)=\left\{\xi \in \mathbf{R}^{n}:|\xi| \leq R\right\}$.

Proof. We assume that $h$ is standardized. Fix $R>0$, and define $g(\xi): \mathbf{R}^{n} \rightarrow[0,1]$ by restricting and truncating $h(\xi)$ :

$$
g(\xi)= \begin{cases}0 & \text { if } \xi \notin B(0, R), \\ \min \{h(\xi), 1\} & \text { if } \xi \in B(0, R) .\end{cases}
$$


Since $h(\xi) \geq g(\xi) \geq 0$ for all $\xi \in \mathbf{R}^{n}$, we have $h * h(\xi) \geq g * g(\xi) \geq 0$ as well. Let $\xi_{0} \in \bar{B}(0, R)$, the closed ball. Since $0<g(\xi) \leq 1$ on $B\left(\xi_{0} / 2, R / 2\right) \subset B(0, R)$, it follows that

$$
g * g\left(\xi_{0}\right) \geq \int_{|\eta|<R / 2} g\left(\frac{\xi_{0}}{2}-\eta\right) g\left(\frac{\xi_{0}}{2}+\eta\right) d \eta>0 .
$$

In other words, for $J=g * g(\bar{B}(0, R))$ we have $J \subset(0, \infty)$. But since $g \in L^{2}\left(\mathbf{R}^{n}\right)$ it follows that $g * g(\xi)$ is continuous on $\mathbf{R}^{n}$, and hence $J$ is compact and connected; i.e., $J$ is a closed subinterval of $(0, \infty)$ that is necessarily bounded away from zero. Then on $\bar{B}(0, R)$ we have

$$
h(\xi) \geq|\xi|^{-\theta} h * h(\xi) \geq|\xi|^{-\theta} g * g(\xi) \geq R^{-\theta} g * g(\xi),
$$

giving that $h(\xi)$ is also bounded away from zero on $\bar{B}(0, R)$.

Corollary 4. If $h \in \mathcal{H}^{\theta}\left(\mathbf{R}^{n}\right), \theta \geq 0$, then for all $\xi_{0} \in \Omega_{h}, \xi_{0} \neq 0$ there exist $\delta=\delta\left(\xi_{0}\right)>0$ and $\varepsilon=\varepsilon\left(\xi_{0}\right)>0$ such that $\inf _{|\eta|<\delta} h\left(\xi_{0}-\eta\right) \geq \varepsilon h\left(\xi_{0}\right)$.

In the following two lemmas $B^{*}(r)=\left\{\xi \in \mathbf{R}^{n}: 0<|\xi|<r\right\}$ denotes the punctured ball of radius $r>0$ in $\mathbf{R}^{n}$ centered at the origin.

Lemma 5. Suppose $h(\xi) \in \mathcal{H}^{\theta}\left(\mathbf{R}^{n}\right)$ with $\theta \geq n / 2$. Then $h(\xi)$ has the following behavior at the origin: either $\liminf _{\xi \rightarrow 0}|\xi|^{\theta} h(\xi)=0$ or $\liminf _{\xi \rightarrow 0}|\xi|^{\theta} h(\xi)=\infty$.

Proof. Assume $h$ is standardized. Suppose that $\liminf _{\xi \rightarrow 0}|\xi|^{\theta} h(\xi)>0$. Then for some $L>0$ there exists a $\delta>0$ such that $|\xi|^{\theta} h(\xi)>L$ for all $\xi \in B^{*}(\delta)$. Then for $\xi \in B^{*}(\delta)$,

$$
|\xi|^{\theta} h(\xi) \geq \int_{|\eta|<\delta / 2} h(\eta) h(\xi-\eta) d \eta \geq \int_{|\eta|<\delta / 2} \frac{L^{2} d \eta}{|\eta|^{\theta}|\xi-\eta|^{\theta}} .
$$

Applying Fatou's Lemma with any convergent sequence $\xi_{n} \rightarrow 0$ gives

$$
\liminf _{\xi_{n} \rightarrow 0}\left|\xi_{n}\right|^{\theta} h\left(\xi_{n}\right) \geq \int_{\left|\eta_{1}\right|<\delta / 2} \liminf _{\xi_{n} \rightarrow 0} \frac{L^{2} d \eta}{|\eta|^{\theta}\left|\xi_{n}-\eta\right|^{\theta}}=\int_{|\eta|<\delta / 2} \frac{L^{2} d \eta}{|\eta|^{2 \theta}}=\infty .
$$

Lemma 6. If $h \in \mathcal{H}^{\theta}\left(\mathbf{R}^{n}\right)$ and $\theta \geq n / 2$, then $\liminf _{\xi \rightarrow 0}|\xi|^{\theta} h(\xi) \neq \infty$.

Proof. Fix $n \geq 1$ and take $\theta \geq n / 2$. Suppose for contradiction that there exists $h \in \mathcal{H}^{\theta}\left(\mathbf{R}^{n}\right)$ with $\liminf _{\xi \rightarrow 0}|\xi|^{\theta} h(\xi)=\infty$. We may assume $h$ is standardized. For $x>0$, let

$$
\rho(x)=\sup \left\{r>0:|\xi|^{\theta} h(\xi)>x \forall \xi \in B^{*}(r)\right\} .
$$

Notice that $\rho$ is a nonincreasing function of $x$ with $\lim _{x \rightarrow \infty} \rho(x)=0$. Furthermore, for any $\xi \in B^{*}(\rho(x) / 2)$,

$$
\begin{aligned}
|\xi|^{\theta} h(\xi) \geq \int_{|\eta|<\rho(x) / 2} h(\eta) h(\xi-\eta) d \eta \geq & \int_{|\eta|<\rho(x) / 2} \frac{x^{2}}{|\eta|^{\theta}|\xi-\eta|^{\theta}} d \eta \\
& \geq \frac{x^{2}}{[\rho(x)]^{\theta}} \int_{|\eta|<\rho(x) / 2} \frac{d \eta}{|\eta|^{\theta}} .
\end{aligned}
$$

The cases $\theta \geq n$ and $n / 2 \leq \theta<n$ are now considered separately. We can easily dispense with the first case. If $\theta \geq n$ and $\rho(x)>0$, then $\int_{|\eta|<\rho(x) / 2}|\eta|^{-\theta} d \eta=\infty$, 
giving $h(\xi) \equiv \infty$ on $B^{*}(\rho(x) / 2)$ and violating Definition 2, Therefore if $h \in \mathcal{H}^{\theta}\left(\mathbf{R}^{n}\right)$ and $\theta \geq n$, then $\liminf _{\xi \rightarrow 0} h(\xi) \neq \infty$.

For $\theta \in[n / 2, n)$, the calculation in (9) gives

$$
|\xi|^{\theta} h(\xi) \geq C_{n, \theta} x^{2}[\rho(x)]^{n-2 \theta} \quad \text { for } \quad \xi \in B^{*}(\rho(x) / 2),
$$

where $C_{n, \theta}=\pi^{n / 2}\left((n-\theta) 2^{n-\theta} \Gamma(n / 2)\right)^{-1}$ depends only on $n$ and $\theta$. This will give a lower bound on the rate at which $|\xi|^{\theta} h(\xi) \rightarrow \infty$ as $|\xi| \rightarrow 0$. In particular, if we define

$$
\lambda(x)=C_{n, \theta}[\rho(x)]^{n-2 \theta},
$$

then inspection of (10) gives

$$
\rho\left(x^{2} \lambda(x)\right) \geq 2^{-1} \rho(x) .
$$

We now define a rapidly increasing sequence $\left\{x_{k}\right\}$ iteratively in a way that allows inequality (11) to control the corresponding decrease in $\rho\left(x_{k}\right)$. This will yield a contradiction with the assumption that $\liminf _{\xi \rightarrow 0}|\xi|^{\theta} h(\xi)=\infty$. First observe that for $n / 2<\theta<n, \lambda(x)$ is a nondecreasing function of $x$ with $\lambda(x) \rightarrow \infty$ as $x \rightarrow \infty$. If $\theta=n / 2$, then $\lambda(x)=C_{n, n / 2}=2(\pi / 2)^{n / 2}(n \Gamma(n / 2))^{-1}$. Accordingly, these two cases are treated separately in defining $\left\{x_{k}\right\}$. For $\theta \in(n / 2, n)$, fix $x_{0} \geq 2$ large enough to also have $\lambda\left(x_{0}\right) \geq 2$ and define $\left\{x_{k}\right\}_{k \geq 1}$ iteratively via

$$
x_{k}=x_{k-1}^{2} \lambda\left(x_{k-1}\right) \quad \text { for } \quad k \geq 1 .
$$

Then $x_{1} \geq 2 x_{0}^{2} \geq 2^{3}$ and by induction,

$$
x_{k} \geq 2 x_{k-1}^{2} \geq 2^{2^{k+1}-1} .
$$

For $\theta=n / 2$, take $x_{0}=\max \left\{2,2\left(C_{n, n / 2}\right)^{-1}\right\}$ and define $\left\{x_{k}\right\}_{k \geq 1}$ iteratively via

$$
x_{k}=C_{n, n / 2} x_{k-1}^{2}=x_{k-1}^{2} \lambda\left(x_{k-1}\right) \text { for } k \geq 1 .
$$

Then $x_{1}=\max \left\{2^{2} C_{n, n / 2}, 2^{2}\left(C_{n, n / 2}\right)^{-1}\right\}$ and by induction,

$$
x_{k}=\max \left\{2^{2^{k}}\left(C_{n, n / 2}\right)^{2^{k}-1}, 2^{2^{k}}\left(C_{n, n / 2}\right)^{-1}\right\} .
$$

Since $\max \left\{\left(C_{n, n / 2}\right)^{2^{k}-1},\left(C_{n, n / 2}\right)^{-1}\right\} \geq 1$, in both cases the sequence satisfies both

$$
x_{k} \geq 2^{2^{k}}, \quad k \geq 0,
$$

and

$$
x_{k}=x_{k-1}^{2} \lambda\left(x_{k-1}\right), \quad k \geq 1 .
$$

In particular, (12) gives $\rho\left(x_{k}\right) \rightarrow 0$ as $k \rightarrow \infty$. On the other hand, (13) combined with (11) controls the rate of decrease of $\rho\left(x_{k}\right)$ via

$$
\rho\left(x_{k}\right) \geq 2^{-1} \rho\left(x_{k-1}\right) \geq 2^{-k} \rho\left(x_{0}\right) .
$$


To conclude the proof, fix $\xi_{0} \in \Omega_{h}, \xi_{0} \neq 0$. By Corollary 4 there exists $\delta, \epsilon>0$ such that $\inf _{|\eta|<\delta} h\left(\xi_{0}-\eta\right) \geq \epsilon h\left(\xi_{0}\right)$. Take $k$ large enough to have $\rho\left(x_{k}\right)<\delta$. Then

$$
\begin{aligned}
\left|\xi_{0}\right|^{\theta} h\left(\xi_{0}\right) \geq & \int_{|\eta|<\rho\left(x_{k}\right)} h(\eta) h\left(\xi_{0}-\eta\right) d \eta \geq \epsilon h\left(\xi_{0}\right) \int_{|\eta|<\rho\left(x_{k}\right)} h(\eta) d \eta \\
& \geq \epsilon h\left(\xi_{0}\right) \int_{|\eta|<\rho\left(x_{k}\right)} \frac{x_{k}}{|\eta|^{\theta}} d \eta=\epsilon h\left(\xi_{0}\right) C_{n, \theta}^{\prime} x_{k} \rho^{n-\theta}\left(x_{k}\right) \\
& \geq \epsilon h\left(\xi_{0}\right) C_{n, \theta}^{\prime} 2^{2^{k}}\left(2^{-k} \rho\left(x_{0}\right)\right)^{n-\theta},
\end{aligned}
$$

where $C_{n, \theta}^{\prime}=2^{n-\theta} C_{n, \theta}$ depends only on $n$ and $\theta$. As the right-hand side of (14) becomes arbitrarily large as $k \rightarrow \infty$, contradicting the finiteness of the left-hand side, we conclude that if $h \in \mathcal{H}^{\theta}\left(\mathbf{R}^{n}\right)$ with $\theta \geq n / 2$, then $\liminf _{\xi \rightarrow 0}|\xi|^{\theta} h(\xi) \neq$ $\infty$.

Proof of Theorem 1. Suppose for contradiction that $h \in \mathcal{H}^{\theta}\left(\mathbf{R}^{n}\right)$ with $\theta \geq n / 2$. Assume that $h$ is standardized. By Lemmas 5 and [6, $\liminf _{\xi \rightarrow 0}|\xi|^{\theta} h(\xi)=0$. Applying Fatou's Lemma we find that

$$
\begin{aligned}
0=\liminf _{\xi \rightarrow 0}|\xi|^{\theta} h(\xi) \geq & \liminf _{\xi \rightarrow 0} \int_{\mathbf{R}^{n}} h(\eta) h(\xi-\eta) d \eta \\
& \geq \int_{\mathbf{R}^{n}} h(\eta) \liminf _{\xi \rightarrow 0} h(\xi-\eta) d \eta=\int_{\mathbf{R}^{n}} h(\eta) \liminf _{\xi \rightarrow-\eta} h(\xi) d \eta,
\end{aligned}
$$

implying that for almost all $\eta \in \mathbf{R}^{n}$,

$$
h(\eta) \liminf _{\xi \rightarrow-\eta} h(\xi)=0 .
$$

In particular, this holds for almost all $\eta \in \mathbf{R}^{n}$ such that $-\eta \in \Omega_{h}$. For any such $-\eta \in \Omega_{h}, h(\xi)$ is bounded away from zero in a neighborhood of $-\eta$ by Corollary 4 , and since $h(\eta)>0$ for all $\eta$,

$$
h(\eta) \liminf _{\xi \rightarrow-\eta} h(\xi)>0 .
$$

This contradicts (15). Therefore if $\theta \geq n / 2$, then $\mathcal{H}^{\theta}\left(\mathbf{R}^{n}\right)=\emptyset$, or equivalently, if $h \in \mathcal{H}^{\theta}\left(\mathbf{R}^{n}\right)$, then $\theta<n / 2$.

\section{Embedding Properties ANd RElation to Koch-Tataru SOlutions}

In this section we discuss properties of majorizing kernels and majorization spaces implied by Theorem 11. One consequence in particular is that for a given majorizing kernel that behaves algebraically at the origin and at infinity we have the continuous embedding $\mathcal{F}_{h} \hookrightarrow P M^{n-\theta}$ (a slight modification is needed if $\theta=0$ ). This is part of a chain of continuous embeddings from $\mathcal{F}_{h}$ up to the spaces $B M O^{-1}$, $B M O_{T}^{-1}$ and $\overline{V M O}^{-1}$, the initial value spaces for the Koch-Tataru solutions of the Navier-Stokes equations. If $\theta=1$, then we have

$$
\mathcal{F}_{h} \hookrightarrow P M^{n-1} \hookrightarrow \dot{B}_{p}^{-1+\frac{n}{p}, \infty} \hookrightarrow B M O^{-1} \quad(n<p<\infty),
$$

and if $0<\theta<1$, then for $0<T<1$ we have

$$
\mathcal{F}_{h} \hookrightarrow P M^{n-\theta} \hookrightarrow \dot{B}_{p}^{-\theta+\frac{n}{p}, \infty} \hookrightarrow B_{p}^{-\theta+\frac{n}{p}, \infty} \hookrightarrow B M O_{T}^{-1} \quad\left(\frac{n}{\theta}<p<\infty\right),
$$


and $\mathcal{F}_{h} \subset \overline{V M O}^{-1}$. Here, as in the sequel, it is convenient to ignore the distinction between spaces of scalar-valued and vector-valued functions. We follow the index convention in 11] for the homogeneous and inhomogeneous Besov spaces, $\dot{B}_{p}^{s, q}$ and $B_{p}^{s, q}$ respectively. Although the successive embeddings after $\mathcal{F}_{h} \hookrightarrow P M^{n-\theta}$ are known, we record them here for completeness and to emphasize how the dichotomy between cases $\theta<1$ and $\theta=1$ aligns with the dichotomy between local and global solutions in the endpoint spaces. Finally, we note that there are majorization spaces $\mathcal{F}_{h} \not \subset P M^{n-\theta}$ that embed further along these chains. Proposition 17illustrates how this can occur. Since the class of majorizing kernels has itself not been completely characterized, we are not able to locate all majorization spaces on scales of classical or better known Banach spaces.

4.1. The endpoint spaces. Recall that Koch and Tataru [13] consider the iteration scheme for solving the mild formulation of the Navier-Stokes equations with initial data in the function space $B M O^{-1}=B M O^{-1}\left(\mathbf{R}^{n}\right)$, which admits a Carleson measure characterization through the norm

$$
\|f\|_{B M O^{-1}}=\sup _{x, R>0}\left(\frac{1}{|B(x, R)|} \int_{0}^{R^{2}} \int_{B(x, R)}\left|e^{t \Delta} f\right|^{2} d y d t\right)^{1 / 2} .
$$

Here $B(x, R)$ denotes the ball of radius $R$ centered at $x \in \mathbf{R}^{n}$, and $|B(x, R)|$ is its Lebesgue measure. Equivalently, $B M O^{-1}$ consists of functions that can be written as the divergence of vector fields whose components belong to $B M O$, the space of functions of bounded mean oscillation.

In [13] it is shown that given sufficiently small initial datum $u_{0} \in B M O^{-1}$, there exists a mild solution of the Navier-Stokes equations issued from $u_{0}$ in the path space $X$ of functions defined on $\mathbf{R}^{n} \times \mathbf{R}^{+}$with norm

$$
\|u\|_{X}=\sup _{t} t^{1 / 2}\|u(t)\|_{L^{\infty}\left(\mathbf{R}^{n}\right)}+\sup _{x, R>0}\left(\frac{1}{|B(x, R)|} \int_{0}^{R^{2}} \int_{B(x, R)}|u|^{2} d y d t\right)^{1 / 2} .
$$

It is also shown that there exists a constant $\varepsilon_{0}$ such that for all $\left\|u_{0}\right\|_{B M O_{T}^{-1}}<\varepsilon_{0}$ there exists a mild solution in the local path space $X_{T}$, defined by the norm

$$
\|u\|_{X_{T}}=\sup _{0<t<T} t^{1 / 2}\|u(t)\|_{L^{\infty}\left(\mathbf{R}^{n}\right)}+\sup _{x, 0<R^{2}<T}\left(\frac{1}{|B(x, R)|} \int_{0}^{R^{2}} \int_{B(x, R)}|u|^{2} d y d t\right)^{\frac{1}{2}} .
$$

Here $B M O_{T}^{-1}$ is defined as $B M O^{-1}$ except that we only consider balls of size $\sqrt{T}$ and smaller:

$$
\|f\|_{B M O_{T}^{-1}}=\sup _{x, 0<R^{2}<T}\left(\frac{1}{|B(x, R)|} \int_{0}^{R^{2}} \int_{B(x, R)}\left|e^{t \Delta} f\right|^{2} d y d t\right)^{1 / 2} .
$$

Finally, $\overline{V M O^{-1}}:=\left\{f \in B M O_{1}^{-1}:\|f\|_{B M O_{T}^{-1}} \rightarrow 0\right.$ as $\left.T \rightarrow 0\right\}$, following the notation of [13]. In [17] and elsewhere, this space is denoted by $v m o^{-1}$.

4.2. Continuous embeddings of majorization spaces. We first define a class of majorizing kernels having certain algebraic growth and decay properties, and then consider continuous embeddings of the associated majorization spaces.

Definition 7. A majorizing kernel $h \in \mathcal{H}^{\theta}\left(\mathbf{R}^{n}\right)$ behaves (algebraically) at the origin as $|\xi|^{-\alpha}$ or blows up (algebraically) at the origin as $|\xi|^{-\alpha}$ if there exists an $\alpha \geq 0$ such that $h(\xi)=O\left(|\xi|^{-\alpha}\right)$ as $\xi \rightarrow 0$ and $|\xi|^{-\alpha}=O(h(\xi))$ as $\xi \rightarrow 0$. 
Definition 8. A majorizing kernel $h \in \mathcal{H}^{\theta}\left(\mathbf{R}^{n}\right)$ behaves (algebraically) at infinity as $|\xi|^{-\omega}$ or decays (algebraically) at infinity as $|\xi|^{-\omega}$ if there exists an $\omega>0$ such that $h(\xi)=O\left(|\xi|^{-\omega}\right)$ as $\xi \rightarrow \infty$ and $|\xi|^{-\omega}=O(h(\xi))$ as $\xi \rightarrow \infty$.

Definition 9. A majorizing kernel has radial algebraic growth and decay if

(1) it behaves algebraically at the origin as $|\xi|^{-\alpha}$ for some $\alpha \geq 0$;

(2) it decays algebraically at infinity as $|\xi|^{-\omega}$ for some $\omega>0$;

(3) it is bounded on the complement of any neighborhood of the origin.

The subclass of $\mathcal{H}^{\theta}\left(\mathbf{R}^{n}\right)$ consisting of those majorizing kernels with radial algebraic growth and decay is denoted $\mathcal{H}_{\alpha, \omega}^{\theta}\left(\mathbf{R}^{n}\right)$.

Note that if $h \in \mathcal{H}_{\alpha, \omega}^{\theta}\left(\mathbf{R}^{n}\right)$, then for any $\lambda>0$ the elements $f \in \mathcal{F}_{h}$ satisfy the scaling relation

$$
C_{\lambda}\|f\|_{\mathcal{F}_{h}} \leq\left\|\lambda^{\theta} f_{\lambda}\right\|_{\mathcal{F}_{h}} \leq C_{\lambda}^{\prime}\|f\|_{\mathcal{F}_{h}},
$$

where $f_{\lambda}(x)=f(\lambda x)$ and constants $C_{\lambda}$ and $C_{\lambda}^{\prime}$ may depend on $\lambda$. On the other hand, this property does not characterize $\mathcal{H}_{\alpha, \omega}^{\theta}\left(\mathbf{R}^{n}\right)$. The majorizing kernels discussed in Proposition 16, for example, satisfy (16) with $C_{\lambda} \equiv C_{\lambda}^{\prime} \equiv 1$ yet do not belong to $\mathcal{H}_{\alpha, \omega}^{\theta}\left(\mathbf{R}^{n}\right)$. Majorization spaces also exist that satisfy (16) only for certain $\lambda$. Taking $h(\xi)=(2 \pi)^{-1}|\xi|^{-1} e^{-|\xi|} \in \mathcal{H}^{1}\left(\mathbf{R}^{3}\right)$, for example, yields a majorization space $\mathcal{F}_{h}$ that satisfies (16) only for $\lambda \leq 1$. In this case, $\lambda\|f\|_{\mathcal{F}_{h}} \leq\left\|\lambda f_{\lambda}\right\|_{\mathcal{F}_{h}} \leq \lambda^{-1}\|f\|_{\mathcal{F}_{h}}$.

Theorem 10. Suppose $h \in \mathcal{H}^{\theta}\left(\mathbf{R}^{n}\right)$ blows up at the origin as $|\xi|^{-\alpha}$. If $0<\theta<n / 2$, then $\alpha \leq n-\theta$, and if $\theta=0$, then $\alpha<n$.

Proof. Assume $h \in \mathcal{H}^{\theta}\left(\mathbf{R}^{n}\right)$ behaves as $|\xi|^{-\alpha}$ at the origin and is standardized. Then $\alpha<n$, lest $h * h(\xi) \equiv \infty$. Suppose for contradiction that $\alpha>n-\theta$. Then $2 \alpha>n$ by Theorem 1. Since $|\xi|^{-\alpha}=O(h(\xi))$ and $h(\xi)=O\left(|\xi|^{-\alpha}\right)$ as $\xi \rightarrow 0$, there exist constants $R>0$ and $C, C_{1}>0$ such that $\sqrt{C_{1}}|\xi|^{-\alpha} \leq h(\xi) \leq C|\xi|^{-\alpha}$ for all $|\xi|<R$. Then for all $|\xi|<R / 3$ we have

$$
C \geq|\xi|^{\alpha-\theta} h * h(\xi) \geq C_{1}|\xi|^{\alpha-\theta} \int_{|\eta|<2 R / 3} \frac{d \eta}{|\xi-\eta|^{\alpha}|\eta|^{\alpha}}
$$

$$
\begin{aligned}
& \geq C_{1}|\xi|^{\alpha-\theta}\left\{\int_{\mathbf{R}^{n}} \frac{d \eta}{|\xi-\eta|^{\alpha}|\eta|^{\alpha}}-\int_{|\eta|>2 R / 3} \frac{d \eta}{|\xi-\eta|^{\alpha}|\eta|^{\alpha}}\right\} \\
& \geq C_{1}\left\{\frac{C_{2}}{|\xi|^{\alpha-(n-\theta)}}-C_{3}|\xi|^{\alpha-\theta}\right\},
\end{aligned}
$$

using the convolution equality $|\xi|^{-\alpha} *|\xi|^{-\alpha}=C_{2}|\xi|^{-(2 \alpha-n)}$ and the estimate

$$
\int_{|\eta|>2 R / 3} \frac{d \eta}{|\xi-\eta|^{\alpha}|\eta|^{\alpha}} \leq C_{3}<\infty
$$

both of which hold if $n / 2<\alpha<n$. Then the right-hand side of (17) tends to $+\infty$ as $\xi \rightarrow 0$, which contradicts the finiteness of the left-hand side. Hence if $h(\xi)$ behaves as $|\xi|^{-\alpha}$ at the origin, then $\alpha \leq n-\theta$.

Theorem 11. If $h(\xi) \in \mathcal{H}^{\theta}\left(\mathbf{R}^{n}\right)$ decays at infinity as $|\xi|^{-\omega}$, then $\omega \geq n-\theta$.

Proof. Assume $h$ behaves as $|\xi|^{-\omega}$ at infinity and is standardized. There exist an $R>0$ and constants $C_{1}, C_{2}$ such that $C_{1}|\xi|^{-\omega} \leq h(\xi) \leq C_{2}|\xi|^{-\omega}$ whenever $|\xi|>R$. 
Using the fact that $2|\xi-\eta| \geq|\eta|$ whenever $|\eta| \geq 2|\xi|$, we have for all $\xi$ such that $|\xi|>R$,

$$
\begin{aligned}
C_{2}|\xi|^{\theta-\omega} \geq|\xi|^{\theta} h(\xi) \geq h * h(\xi) \geq & \int_{|\eta| \geq 2|\xi|} h(\xi-\eta) h(\eta) d \eta \\
& \geq 2^{-\omega} C_{1}^{2} \int_{|\eta| \geq 2|\xi|}|\eta|^{-2 \omega} d \eta=C|\xi|^{n-2 \omega} .
\end{aligned}
$$

This implies $2 \omega>n$ and $|\xi|^{n-2 \omega}=O\left(|\xi|^{\theta-\omega}\right)$ as $\xi \rightarrow \infty$; hence $\omega \geq \max \{n / 2, n-\theta\}$. The maximum here is superfluous by Theorem 1, and we have $\omega \geq n-\theta>n / 2$.

Given Banach spaces $X$ and $Y$ we write $X \hookrightarrow Y$ to denote the continuous embedding of $X$ into $Y$.

Theorem 12. Suppose $h \in \mathcal{H}_{\alpha, \omega}^{\theta}\left(\mathbf{R}^{n}\right), \theta<n / 2$ and $n \geq 2$. If $\theta=1$, then $\mathcal{F}_{h} \hookrightarrow P M^{n-1} \hookrightarrow B M O^{-1}$. If $0<\theta<1$, then $\mathcal{F}_{h} \hookrightarrow P M^{n-\theta} \hookrightarrow B M O_{T}^{-1}$ for all $T>0$.

Remark 13. The following intermediate embeddings are also known: for $p \in(n, \infty)$, $n \geq 2$,

$$
P M^{n-1} \hookrightarrow \dot{B}_{p}^{-1+\frac{n}{p}, \infty} \hookrightarrow B M O^{-1} .
$$

See e.g. [8, p. 267], [1, p. 228], respectively. For $p \in(n / \theta, \infty), n \geq 2,0<\theta<1$ and $0<T \leq 1$, we also have

$$
P M^{n-\theta} \hookrightarrow \dot{B}_{p}^{-\theta+\frac{n}{p}, \infty} \hookrightarrow B_{p}^{-\theta+\frac{n}{p}, \infty} \hookrightarrow B M O_{T}^{-1}
$$

through a suitable modification of [8, Lemma 7.1] and [13, Remark 4.2f.]. In addition, $P M^{n-\theta} \subset \overline{V M O}^{-1}$, since the constant of the final embedding is proportional to $T^{(1-\theta) / 2}$.

Recall that for the pair $\left\{L^{q}=L^{q}\left(\mathbf{R}^{n}\right), L^{r}=L^{r}\left(\mathbf{R}^{n}\right)\right\}, 1 \leq q, r \leq \infty$, the set $L^{q}+L^{r}=\left\{f_{q}+f_{r}: f_{q} \in L^{q}, f_{r} \in L^{r}\right\}$ becomes a Banach space when equipped with the norm

$$
\|f\|_{L^{q}+L^{r}}=\inf \left\{\left\|f_{q}\right\|_{L^{q}}+\left\|f_{r}\right\|_{L^{r}}: f=f_{q}+f_{r}\right\} .
$$

Proof of Theorem 12. We first consider simultaneously $P M^{n-1} \hookrightarrow B M O^{-1}(n \geq$ 2) and $P M^{n-\theta} \hookrightarrow B M O_{T}^{-1}(n \geq 2,0<\theta<1)$. Recall that $f_{\lambda}(x)=f(\lambda x)$. By scaling we have

$$
\frac{1}{\left|B\left(x_{0}, \lambda\right)\right|} \int_{0}^{\lambda^{2}} \int_{B\left(x_{0}, \lambda\right)}\left|e^{t \Delta} f\right|^{2} d y d t=\frac{\lambda^{n+2-2 \theta}}{\left|B\left(x_{0}, \lambda\right)\right|} \int_{0}^{1} \int_{B\left(\lambda^{-1} x_{0}, 1\right)}\left|e^{t \Delta} \lambda^{\theta} f_{\lambda}\right|^{2} d y d t .
$$

The following estimate holds for all $f \in P M^{n-\theta}(n \geq 2,0<\theta \leq 1)$ :

$$
\left(\int_{B\left(x_{0}, 1\right)}\left|e^{t \Delta} f\right|^{2} d y\right)^{\frac{1}{2}} \leq\left\|f^{(2)}\right\|_{L^{2}}+\left|B\left(x_{0}, 1\right)\right|^{\frac{1}{2}}\left\|f^{(\infty)}\right\|_{L^{\infty}} \leq C\|f\|_{P M^{n-\theta}}\left\|h^{\star}\right\|_{L^{1}+L^{2}} .
$$

Here $h^{\star}=|\xi|^{-(n-\theta)} \in L^{1}+L^{2}$ and $f=f^{(2)}+f^{(\infty)} \in L^{2}+L^{\infty}$. Applying this estimate to the right-hand side of (18) along with the scaling relation $\left\|\lambda^{\theta} f_{\lambda}\right\|_{P M^{n-\theta}}=\|f\|_{P M^{n-\theta}}$ gives

$$
\begin{aligned}
& \|f\|_{B M O_{T}^{-1}} \leq C_{\theta} T^{(1-\theta) / 2}\|f\|_{P M^{n-\theta}} \quad(0<\theta<1), \\
& \|f\|_{B M O^{-1}} \leq C_{\theta}\|f\|_{P M^{n-1}} \quad(\theta=1),
\end{aligned}
$$


where $C_{\theta}=C\left\|h^{\star}\right\|_{L^{1}+L^{2}}$ depends on $\theta$. Now suppose $h \in \mathcal{H}_{\alpha, \omega}^{\theta}\left(\mathbf{R}^{n}\right), \theta<n / 2$, $n \geq 2$ and $0<\theta \leq 1$. Theorems 10 and 11 imply $\sup _{\xi \in \mathbf{R}^{n}}|\xi|^{n-\theta} h(\xi)<\infty$, and then $\mathcal{F}_{h} \hookrightarrow P M^{n-\theta}$ follows.

Remark 14. Theorems 10 and 11 also imply that if $h \in \mathcal{H}_{\alpha, \omega}^{0}\left(\mathbf{R}^{n}\right)$, then there exists a constant $\sigma$ (depending on $h$ ) with $0<\sigma<1$ such that $\mathcal{F}_{h} \hookrightarrow P M^{n-\sigma}$.

The next theorem deals with inclusions $\mathcal{F}_{h} \subset \overline{V M O}^{-1}$, where the latter space plays a role in the local solutions analyzed in [13]. We write $\mathbf{1}[\cdot]$ for the indicator function and $\mathcal{F}^{-1}$ for the inverse Fourier transform.

Theorem 15. Suppose $h \in \mathcal{H}_{\alpha, \omega}^{\theta}\left(\mathbf{R}^{n}\right), n \geq 2$. If $0<\theta<1$, then there exists a constant $C_{\theta}$ (which may depend on $\theta$ ) such that for all $f \in \mathcal{F}_{h}, T>0$,

$$
\|f\|_{B M O_{T}^{-1}} \leq C_{\theta} T^{(1-\theta) / 2}\|f\|_{\mathcal{F}_{h}}
$$

hence $\mathcal{F}_{h} \subset \overline{V M O}^{-1}$. If $\theta=0$, then there exist constants $C, k_{1}$, and $k_{2}$ such that

$$
\|f\|_{B M O_{T}^{-1}} \leq \begin{cases}C T^{1 / 2}\|f\|_{\mathcal{F}_{h}} & \text { if } \omega>n, \\ \left(k_{1} T^{1 / 2}+k_{2} T^{1 / 2} \log T^{-1 / 2}\right)\|f\|_{\mathcal{F}_{h}} & \text { if } \omega=n\end{cases}
$$

for all $f \in \mathcal{F}_{h}, 0<T \leq 1 / e^{2}$; hence $\mathcal{F}_{h} \subset \overline{V M O}^{-1}$.

Proof. The proof of Theorem 12 implies (19) for $0<\theta<1$. If $\theta=0$ and $\omega>n$, then $h \in L^{1}$. Then for all $f \in \mathcal{F}_{h}, \lambda>0$, we have

$$
\left(\int_{B\left(\lambda^{-1} x_{0}, 1\right)}\left|e^{t \Delta} f_{\lambda}\right|^{2} d y\right)^{\frac{1}{2}} \leq\left|B\left(\lambda^{-1} x_{0}, 1\right)\right|^{\frac{1}{2}}\left\|f_{\lambda}\right\|_{L^{\infty}} \leq C\|f\|_{L^{\infty}} \leq C\|f\|_{\mathcal{F}_{h}}\|h\|_{L^{1}} .
$$

Applying this to (18) gives the first part of (20). If $\theta=0$ and $\omega=n$ we let $f_{\lambda}=f_{\lambda}^{(2)}+f_{\lambda}^{(\infty)} \in L^{2}+L^{\infty}$ where

$$
f_{\lambda}^{(2)}=f_{\lambda}^{(2, R)}=\mathcal{F}^{-1}\left(\widehat{f_{\lambda}} \mathbf{1}[|\xi| \geq R]\right), \quad f_{\lambda}^{(\infty)}=f_{\lambda}^{(\infty, R)}=\mathcal{F}^{-1}\left(\widehat{f_{\lambda}} \mathbf{1}[|\xi|<R]\right),
$$

and $R$ is chosen so that $C_{1}|\xi|^{-n} \mathbf{1}_{[|\xi| \geq R]} \leq h(\xi) \mathbf{1}_{[|\xi| \geq R]} \leq C_{2}|\xi|^{-n} \mathbf{1}_{[|\xi| \geq R]}$. Then if $\lambda<1$ we have

$$
\begin{aligned}
& \left(\int_{B\left(\lambda^{-1} x_{0}, 1\right)}\left|e^{t \Delta} f_{\lambda}^{(2)}\right|^{2} d y\right)^{\frac{1}{2}} \leq C \sup _{|\xi|>R} \frac{\lambda^{-n} \hat{f}\left(\lambda^{-1} \xi\right)}{\lambda^{-n} h\left(\lambda^{-1} \xi\right)} \sup _{|\xi|>R} \frac{\lambda^{-n} h\left(\lambda^{-1} \xi\right)}{h(\xi)}\left\|h \mathbf{1}_{[|\xi| \geq R]}\right\|_{L^{2}} \\
& \leq C \frac{C_{2}}{C_{1}}\|f\|_{\mathcal{F}_{h}}\left\|h \mathbf{1}_{[|\xi|>R]}\right\|_{L^{2}} \quad(\lambda<1) .
\end{aligned}
$$

In addition, there exist constants $c_{1}$ and $c_{2}$ such that if $\lambda<1$, we have

$$
\begin{aligned}
&\left(\int_{B\left(\lambda^{-1} x_{0}, 1\right)}\left|e^{t \Delta} f_{\lambda}^{(\infty)}\right|^{2} d y\right)^{\frac{1}{2}} \leq\left|B\left(\lambda^{-1} x_{0}, 1\right)\right|^{\frac{1}{2}}\left\|f_{\lambda}^{(\infty)}\right\|_{L^{\infty}} \\
& \leq C\|f\|_{\mathcal{F}_{h}} \int_{\mathbf{R}^{n}} h(\xi) \mathbf{1}[\lambda|\xi|<R] d \xi \\
& \leq C\left(c_{1}+c_{2} \log \lambda^{-1}\right)\|f\|_{\mathcal{F}_{h}} \quad(\lambda<1) .
\end{aligned}
$$


Applying these estimates to (18) gives the second part of (20) provided $T \leq e^{-2}$, which insures that $\lambda \log \lambda^{-1}$ is increasing on $\left(0, T^{1 / 2}\right]$. Finally, (20) is sufficient to conclude that $\mathcal{F}_{h} \subset \overline{V M O}^{-1}$, since similar considerations imply $\mathcal{F}_{h} \subset B M O_{1}^{-1}$.

4.3. Further properties. Theorems 12 and 15 use the fact that if $h \in \mathcal{H}_{\alpha, \omega}^{\theta}\left(\mathbf{R}^{n}\right)$, then $h \in L^{1}+L^{2}$. Not all majorizing kernels share this property. Proposition 16 provides a class of counterexamples, making use of the following criterion for $L^{q}+L^{r}$ : a measurable function $f$ defined on $\mathbf{R}^{n}$ belongs to $L^{q}+L^{r}, 1 \leq q<r \leq \infty$, if and only if for all $M>0, f \mathbf{1}_{[|f| \geq M]} \in L^{q}$ and $f \mathbf{1}_{[|f| \leq M]} \in L^{r}$.

Proposition 16. Let $n \geq 2, k \geq 2$ and $\vartheta<n / 2$ and partition the coordinate index set of $\mathbf{R}^{n}$ into $k$ blocks: $\{1, \ldots, n\}=I_{1} \cup \cdots \cup I_{k},\left|I_{i}\right|=d_{i}, \sum d_{i}=n$. Define $h(\xi)$ on $\mathbf{R}^{n}$ by

$$
h(\xi)=\prod_{i=1}^{k} r_{i}^{-\left(d_{i}-\theta_{i}\right)}, \quad r_{i}^{2}=\sum_{j \in I_{i}} \xi_{j}^{2}, \quad \xi=\left(\xi_{1}, \ldots, \xi_{n}\right)
$$

where $\sum_{i=1}^{k} \theta_{i}=\vartheta, 0<\theta_{i}<d_{i} / 2$. Then $h \in \mathcal{H}^{\vartheta}\left(\mathbf{R}^{n}\right)$ and $h \notin L^{1}+L^{2}$.

Proof. For $h$ as defined above and any set $A \subset \mathbf{R}^{+}$, we have

$$
\int_{\mathbf{R}^{n}} h(\xi) \mathbf{1}[h \in A] d \xi=C \int_{0}^{\infty} \ldots \int_{0}^{\infty}\left(\prod_{i=1}^{k} r_{i}^{\theta_{i}-1}\right) \mathbf{1}\left[\prod_{i=1}^{k} r_{i}^{-\left(d_{i}-\theta_{i}\right)} \in A\right] d r_{1} \cdots d r_{k}
$$

where $C$ is a constant depending only on $\left(d_{1}, \ldots, d_{k}\right)$. In particular,

$$
\int_{\mathbf{R}^{n}} h(\xi) \mathbf{1}[h \geq 1] d \xi=C \int_{0}^{\infty} \ldots \int_{0}^{\infty}\left(\prod_{i=1}^{k} r_{i}^{\theta_{i}-1}\right) \mathbf{1}\left[\prod_{i=1}^{k} r_{i}^{d_{i}-\theta_{i}} \leq 1\right] d r_{1} \cdots d r_{k}=\infty
$$

so $h \notin L^{1}+L^{2}$. On the other hand, $h$, defined on $\mathbf{R}^{n} \simeq \mathbf{R}^{d_{1}} \times \cdots \times \mathbf{R}^{d_{k}}$, has the form $h=\prod_{i}^{k} h_{i}$ with each $h_{i} \in \mathcal{H}^{\theta_{i}}\left(\mathbf{R}^{d_{i}}\right)$, so

$$
h * h(\xi) \leq B_{1} \cdots B_{k}\left|r_{1}\right|^{\theta_{1}} \cdots\left|r_{k}\right|^{\theta_{k}} h(\xi) \leq B_{1} \cdots B_{k}|\xi|^{\vartheta} h(\xi)
$$

and $h \in \mathcal{H}^{\vartheta}\left(\mathbf{R}^{n}\right)$.

The next proposition shows how $\mathcal{F}_{h} \hookrightarrow B_{p}^{-\theta+\frac{n}{p}, \infty}, \mathcal{F}_{h} \not \subset P M^{n-\theta}$ can occur. Following [8, Lemma 7.1], we use the heat semigroup characterization of the spaces $\dot{B}_{p}^{-\alpha, \infty}, \alpha>0$, via the norm $\|f\|_{\dot{B}_{p}^{-\alpha, \infty}}=\sup _{t \geq 0} t^{\alpha / 2}\left\|e^{t \Delta} f\right\|_{L^{p}}$ (see [1, p. 72]).

Proposition 17. Let $n \geq 2, \vartheta<n / 2$, and suppose $h \in \mathcal{H}^{\vartheta}\left(\mathbf{R}^{n}\right)$ is defined as in Proposition 16. Then for all $p>n / \vartheta$, we have $\mathcal{F}_{h} \hookrightarrow \dot{B}_{p}^{-\vartheta+(n / p), \infty}$, but for $a \geq 0$, $\mathcal{F}_{h} \not \subset P M^{a}$. 
Proof. First $\mathcal{F}_{h} \not \subset P M^{a}, a \geq 0$, since $\Omega_{h} \neq \mathbf{R}^{n} \backslash\{0\}$. Let $n / \vartheta<p<\infty$ and set $q^{-1}+p^{-1}=1$ so that $1<q<n /(n-\vartheta)$. By the Hausdorff-Young inequality, for all $f \in \mathcal{F}_{h}, t>0$, we have

$$
\begin{aligned}
\left\|e^{t \Delta} f\right\|_{L^{p}}^{q} \leq & C \int_{\mathbf{R}^{n}}\left|e^{-t|\xi|^{2}} \hat{f}(\xi)\right|^{q} d \xi \\
& \leq C\left(\sup _{\xi \in \mathbf{R}^{n}} \frac{|\hat{f}(\xi)|}{h(\xi)}\right)^{q} \prod_{i=1}^{k}\left(\int_{0}^{\infty} r^{d_{i}-\left(d_{i}-\theta_{i}\right) q} e^{-q r^{2} t} \frac{d r}{r}\right) \\
& \leq C\|f\|_{\mathcal{F}_{h}}^{q} \prod_{i=1}^{k} t^{-\left(d_{i}-\left(d_{i}-\theta_{i}\right) q\right) / 2}=C\|f\|_{\mathcal{F}_{h}}^{q} t^{(-n+(n-\vartheta) q) / 2} .
\end{aligned}
$$

Then $\sup _{t>0} t^{(\vartheta-n / p) / 2}\left\|e^{t \Delta} f\right\|_{L^{p}} \leq C\|f\|_{\mathcal{F}_{h}}$.

\section{ACKNOWLEDGEMENTS}

This work grew out of the collaborative research effort of the authors of 3 ] funded by the U.S. National Science Foundation. We are grateful to the anonymous referees for carefully reading a previous version of the paper and for making suggestions that resulted in significant improvements.

\section{REFERENCES}

1. Hajer Bahouri, Jean-Yves Chemin, and Raphaël Danchin. Fourier analysis and nonlinear partial differential equations, volume 343 of Grundlehren der Mathematischen Wissenschaften [Fundamental Principles of Mathematical Sciences]. Springer, Heidelberg, 2011. MR 2768550 (2011m:35004)

2. Yu. Yu. Bakhtin, E. I. Dinaburg, and Ya. G. Sină̌. On solutions with infinite energy and enstrophy of the Navier-Stokes system. Uspekhi Mat. Nauk, 59(6(360)):55-72, 2004. MR2138467 (2006g:35202)

3. Rabi N. Bhattacharya, Larry Chen, Scott Dobson, Ronald B. Guenther, Chris Orum, Mina Ossiander, Enrique Thomann, and Edward C. Waymire. Majorizing kernels and stochastic cascades with applications to incompressible Navier-Stokes equations. Trans. Amer. Math. Soc., 355(12):5003-5040 (electronic), 2003. MR.1997593(2004k:35294)

4. Rabi N. Bhattacharya, Larry Chen, Ronald B. Guenther, Chris Orum, Mina Ossiander, Enrique Thomann, and Edward C. Waymire. Semi-Markov cascade representations of local solutions to 3-d incompressible Navier-Stokes. In Probability and Partial Differential Equations in Modern Applied Mathematics, volume 140 of The IMA Volumes in Mathematics and its Applications. Springer-Verlag, New York, 2005.

5. D. Blömker, M. Romito, and R. Tribe. A probabilistic representation for the solutions to some non-linear PDEs using pruned branching trees. Ann. Inst. H. Poincaré Probab. Statist., 43(2):175-192, 2007. MR2303118(2008c:60083)

6. Marco Cannone. Viscous flows in Besov spaces. In Advances in mathematical fluid mechanics (Paseky, 1999), pages 1-34. Springer, Berlin, 2000. MR.1863208 (2003f:76027)

7. Marco Cannone. Harmonic analysis tools for solving the incompressible Navier-Stokes equations. In Handbook of mathematical fluid dynamics. Vol. III, pages 161-244. North-Holland, Amsterdam, 2004. MR2099035 (2006c:35216)

8. Marco Cannone and Grzegorz Karch. Smooth or singular solutions to the Navier-Stokes system? J. Differential Equations, 197(2):247-274, 2004. MR2034160 (2005g:35228)

9. Larry Chen, Scott Dobson, Ronald Guenther, Chris Orum, Mina Ossiander, Enrique Thomann, and Edward Waymire. On Itô's complex measure condition. In Probability, statistics and their applications: papers in honor of Rabi Bhattacharya, volume 41 of IMS Lecture Notes Monogr. Ser., pages 65-80. Inst. Math. Statist., Beachwood, OH, 2003. MR1999415 (2004f:60163) 
10. Larry Chen, Ronald B. Guenther, Sun-Chul Kim, Enrique A. Thomann, and Edward C. Waymire. A rate of convergence for the LANS $\alpha$ regularization of Navier-Stokes equations. J. Math. Anal. Appl., 348(2):637-649, 2008. MR2445766 (2009i:76044)

11. Loukas Grafakos. Modern Fourier analysis, volume 250 of Graduate Texts in Mathematics. Springer, New York, second edition, 2009. MR2463316 (2011d:42001)

12. Massimiliano Gubinelli. Rooted trees for 3D Navier-Stokes equation. Dyn. Partial Differ. Equ., 3(2):161-172, 2006. MR2227041 (2007i:35179)

13. Herbert Koch and Daniel Tataru. Well-posedness for the Navier-Stokes equations. Adv. Math., 157(1):22-35, 2001. MR.1808843 (2001m:35257)

14. Y. Le Jan and A. S. Sznitman. Stochastic cascades and 3-dimensional Navier-Stokes equations. Probab. Theory Related Fields, 109(3):343-366, 1997. MR 1481125 (98j:35144)

15. P. G. Lemarié-Rieusset. Recent developments in the Navier-Stokes problem, volume 431 of Chapman $8 \mathrm{Hall} / \mathrm{CRC}$ Research Notes in Mathematics. Chapman \& Hall/CRC, Boca Raton, FL, 2002. MR1938147 (2004e:35178)

16. Yves Meyer. Oscillating patterns in some nonlinear evolution equations. In Mathematical foundation of turbulent viscous flows, volume 1871 of Lecture Notes in Math., pages 101-187. Springer, Berlin, 2006. MR2196363(2007a:35069)

17. Hideyuki Miura. Remark on uniqueness of mild solutions to the Navier-Stokes equations. J. Funct. Anal., 218(1):110-129, 2005. MR2101216 (2005h:35272)

18. Francesco Morandin. A resummed branching process representation for a class of nonlinear ODEs. Electron. Comm. Probab., 10:1-6 (electronic), 2005. MR2119148 (2006a:60161)

19. Mina Ossiander. A probabilistic representation of solutions of the incompressible NavierStokes equations in $\mathbf{R}^{3}$. Probab. Theory Related Fields, 133(2):267-298, 2005. MR2198702 (2007f:76159)

20. Ya. G. Sinal̆. A diagrammatic approach to the 3D Navier-Stokes system. Uspekhi Mat. Nauk, 60(5(365)):47-70, 2005. English translation in: Russian Math. Surveys 60 (2005), no. 5, 849873. MR2195676 (2007e:76055)

21. Ya. G. Sinai. On local and global existence and uniqueness of solutions of the 3D Navier-Stokes system on $\mathbf{R}^{3}$. In Perspectives in analysis, volume 27 of Math. Phys. Stud., pages 269-281. Springer, Berlin, 2005. MR2215729(2007b:35254)

22. Yakov Sinai. Power series for solutions of the 3D-Navier-Stokes system on $\mathbf{R}^{3}$. J. Stat. Phys., 121(5-6):779-803, 2005. MR2192534(2009d:76039)

Department of Mathematics, University of Utah, 155 S. 1400 E., Room 233, Salt Lake Сity, UTAн 84112-0090

E-mail address: orum@math.utah.edu

Current address: Mathematics Program, Eastern Oregon University, La Grande, Oregon 97850

E-mail address: orum@math.utah.edu

Department of Mathematics, Oregon State University, Corvallis, Oregon 97331-4605

E-mail address: ossiand@math.oregonstate.edu 